\title{
Time-Dependent Flow with Convective Heat Transfer through a Curved Square Duct with Large Pressure Gradient
}

\author{
Rabindra Nath Mondal1*, Md. Nurul Amin Helal', Poly Rani Shaha1, Nayan Kumar Poddar ${ }^{1}$ \\ ${ }^{1}$ Department of Mathematics, Jagannath University, Dhaka, Bangladesh \\ ${ }^{2}$ Additional Director (Education), Training Directorate, BGB Head Quarter, Pilkhana, Dhaka, Bangladesh \\ Email: ${ }^{*}$ rnmondal71@yahoo.com
}

Received 10 June 2015; accepted 22 September 2015; published 25 September 2015

Copyright (C) 2015 by authors and Scientific Research Publishing Inc.

This work is licensed under the Creative Commons Attribution International License (CC BY). http://creativecommons.org/licenses/by/4.0/

c. (i) Open Access

\section{Abstract}

A numerical study is presented for the fully developed two-dimensional laminar flow of viscous incompressible fluid through a curved square duct for the constant curvature $\delta=0.1$. In this paper, a spectral-based computational algorithm is employed as the principal tool for the simulations, while a Chebyshev polynomial and collocation method as secondary tools. Numerical calculations are carried out over a wide range of the pressure gradient parameter, the Dean number, $100 \leq D n \leq$ 3000 for the Grashof number, Gr, ranging from 100 to 2000 . The outer wall of the duct is treated heated while the inner wall cooled, the top and bottom walls being adiabatic. The main concern of the present study is to find out the unsteady flow behavior i.e. whether the unsteady flow is steady-state, periodic, multi-periodic or chaotic, if $D$ or $G r$ is increased. It is found that the unsteady flow is periodic for $D n=1000$ at $G r=100$ and 500 and at $D n=2000, G r=2000$ but steady-state otherwise. It is also found that for large values of $D n$, for example $D n=3000$, the unsteady flow undergoes in the scenario "periodic $\rightarrow$ chaotic $\rightarrow$ periodic", if $G r$ is increased. Typical contours of secondary flow patterns and temperature profiles are also obtained, and it is found that the unsteady flow consists of single-, two-, three- and four-vortex solutions. The present study also shows that there is a strong interaction between the heating-induced buoyancy force and the centrifugal force in a curved square passage that stimulates fluid mixing and consequently enhance heat transfer in the fluid.

\section{Keywords}

Curved Square Duct, Secondary Flow, Time-Evolution, Periodic Solution, Chaos

\footnotetext{
${ }^{*}$ Corresponding author.
} vective Heat Transfer through a Curved Square Duct with Large Pressure Gradient. Open Journal of Fluid Dynamics, 5, 238-255. http://dx.doi.org/10.4236/ojfd.2015.53026 


\section{Introduction}

Fluid flow and heat transfer in curved ducts have been studied for a long time because of their fundamental importance in engineering and industrial applications. Today, the flows in curved non-circular ducts are of increasing importance in micro-fluidics, where lithographic methods typically produce channels of square or rectangular cross-section. These channels are extensively used in many engineering applications, such as in turbo-machinery, refrigeration, air conditioning systems, heat exchangers, rocket engine, internal combustion engines and blade-to-blade passages in modern gas turbines. In a curved duct, centrifugal forces are developed in the flow due to channel curvature causing a counter rotating vortex motion applied on the axial flow through the channel. This creates characteristics spiraling fluid flow in the curved passage known as secondary flow. At a certain critical flow condition and beyond, additional pairs of counter rotating vortices appear on the outer concave wall of curved fluid passages which are known as Dean vortices, in recognition of the pioneering work in this field by Dean [1]. After that, many theoretical and experimental investigations have been done; for instance, the articles by Berger et al. [2], Nandakumar and Masliyah [3], and Ito [4] may be referenced.

One of the interesting phenomena of the flow through a curved duct is the bifurcation of the flow because generally there exist many steady solutions due to channel curvature. Studies of the flow through a curved duct have been made, experimentally or numerically, for various shapes of the cross section by many authors. However, an extensive treatment of the bifurcation structure of the flow through a curved duct of rectangular cross section was presented by Winters [5], Daskopoulos and Lenhoff [6] and Mondal [7].

Unsteady flows by time evolution calculation of curved duct flows was first initiated by Yanase and Nishiyama [8] for a rectangular cross section. In that study they investigated unsteady solutions for the case where dual solutions exist. The time-dependent behavior of the flow in a curved rectangular duct of large aspect ratio was investigated, in detail, by Yanase et al. [9] numerically. They performed time-evolution calculations of the unsteady solutions with and without symmetry condition and found that periodic oscillations appear with symmetry condition while aperiodic time variation without symmetry condition. Wang and Yang [10] [11] performed numerical as well as experimental investigation on fully developed periodic oscillation in a curved square duct. Flow visualization in the range of Dean numbers from 50 to 500 was carried out in their experiment. Recently, Yanase et al. [12] performed numerical investigation of isothermal and non-isothermal flows through a curved rectangular duct and addressed the time-dependent behavior of the unsteady solutions. In the succeeding paper, Yanase et al. [13] extended their work for moderate Grashof numbers and studied the effects of secon-dary flows on convective heat transfer. Recently, Mondal et al. [14] [15] performed numerical prediction of the unsteady solutions by time-evolution calculations for the flow through a curved square duct and discussed the transitional behavior of the unsteady solutions.

One of the most important applications of curved duct flow is to enhance the thermal exchange between two sidewalls, because it is possible that the secondary flow may convey heat and then increases heat flux between two sidewalls. Chandratilleke and Nursubyakto [16] presented numerical calculations to describe the secondary flow characteristics in the flow through curved ducts of aspect ratios ranging from 1 to 8 that were heated on the outer wall, where they studied for small Dean numbers and compared the numerical results with their experimental data. Yanase et al. [13] studied time-dependent behavior of the unsteady solutions for curved rectangular duct flow and showed that secondary flows enhance heat transfer in the flow. Mondal et al. [17] performed numerical prediction of the unsteady solutions by time-evolution calculations of the thermal flow through a curved square duct and studied convective heat transfer in the flow. Recently Norouzi et al. [18] [19] investigated fully developed flow and heat transfer of viscoelastic materials in curved square ducts under constant heat flux. Very recently, Chandratilleke and Narayanaswamy [20] numerically studied vortex structure-based analysis of laminar flow and thermal characteristics in curved square and rectangular ducts. To the best of the authors' knowledge, however, there has not yet been done any substantial work studying the transitional behavior of the unsteady solutions for thermal flows through a curved square duct for combined effects of large Grashof number and large Dean number, which has very practical applications in fluids engineering, for example, in internal combustion engine, gas turbines etc. Thus from the scientific as well as engineering point of view it is quite interesting to study the unsteady flow behavior in the presence of strong buoyancy and centrifugal forces. Keeping this issue in mind, in this paper, a comprehensive numerical study is presented for fully developed two-dimensional (2D) flow of viscous incompressible fluid through a curved square duct and studied effects of secondary flows on convective heat transfer in the flow. 


\section{Mathematical Formulations}

Consider an incompressible viscous fluid streaming through a curved duct with square cross section whose width or height is $2 d$. The coordinate system is shown in Figure 1. It is assumed that the temperature of the outer wall is $T_{0}+\Delta T$ and that of the inner wall is $T_{0}-\Delta T$, where $\Delta T>0$. The $x, y$, and $z$ axes are taken to be in the horizontal, vertical, and axial directions, respectively. It is assumed that the flow is uniform in the axial direction, and that it is driven by a constant pressure gradient $G$ along the center-line of the duct, i.e. the main flow in the axial direction as shown in Figure 1. The variables are non-dimensionalized by using the representative length $d$ and the representative velocity $U_{0}=v / d$.

We introduce the non-dimensional variables defined as

$$
\begin{gathered}
u=\frac{u^{\prime}}{U_{0}}, \quad v=\frac{v^{\prime}}{U_{0}}, \quad w=\frac{\sqrt{2 \delta}}{U_{0}} w^{\prime}, \quad x=\frac{x^{\prime}}{d}, \quad \bar{y}=\frac{y^{\prime}}{d}, \quad z=\frac{z^{\prime}}{d} \\
T=\frac{T^{\prime}}{\Delta T}, \quad t=\frac{U_{0}}{d} t^{\prime}, \quad \delta=\frac{d}{L}, \quad P=\frac{P^{\prime}}{\rho U_{0}^{2}}, \quad G=-\frac{\partial P^{\prime}}{\partial z^{\prime}},
\end{gathered}
$$

where, $u, v$ and $w$ are the non-dimensional velocity components in the $x, y$ and $z$ directions, respectively; $t$ is the non-dimensional time, $P$ the non-dimensional pressure, $\delta$ the non-dimensional curvature, and temperature is non-dimensionalized by $\Delta T$. Henceforth, all the variables are nondimensionalized if not specified. The stream function $\psi$ is introduced in the $x$ - and $y$-directions as

$$
u=\frac{1}{1+\delta x} \frac{\partial \psi}{\partial y}, \quad v=-\frac{1}{1+\delta x} \frac{\partial \psi}{\partial x} .
$$

Then the basic equations for $w, \psi$ and $T$ are derived from the Navier-Stokes equations and the energy equation under the Boussinesq approximation as,

$$
(1+\delta x) \frac{\partial w}{\partial t}+\frac{\partial(w, \psi)}{\partial(x, y)}-D n+\frac{\delta^{2} w}{1+\delta x}=(1+\delta x) \Delta_{2} w-\frac{\delta}{(1+\delta x)} \frac{\partial \psi}{\partial y} w+\delta \frac{\partial w}{\partial x},
$$

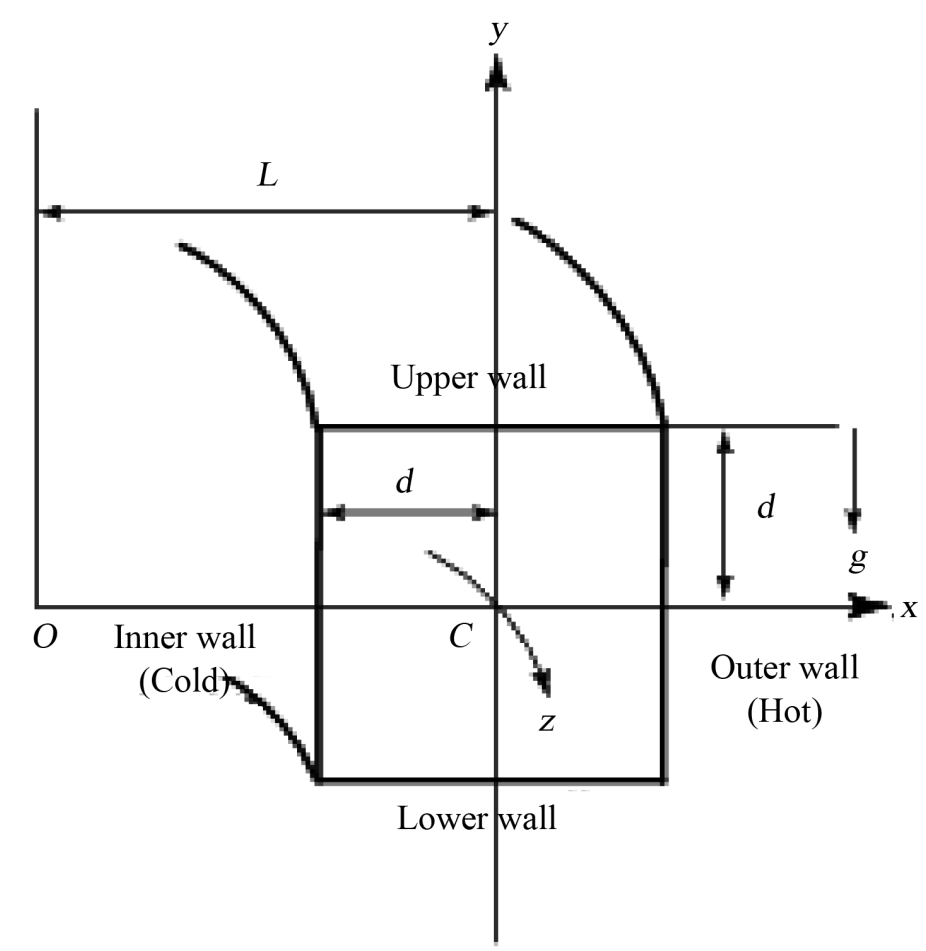

Figure 1. Coordinate system of the curved square duct. 


$$
\begin{aligned}
\left(\Delta_{2}-\frac{\delta}{1+\delta x} \frac{\partial}{\partial x}\right) \frac{\partial \psi}{\partial t}= & -\frac{1}{(1+\delta x)} \frac{\partial\left(\Delta_{2} \psi, \psi\right)}{\partial(x, y)}+\frac{\delta}{(1+\delta x)^{2}}\left[\frac{\partial \psi}{\partial y}\left(2 \Delta_{2} \psi-\frac{3 \delta}{1+\delta x} \frac{\partial \psi}{\partial x}+\frac{\partial^{2} \psi}{\partial x^{2}}\right)\right. \\
& -\frac{\partial \psi}{\partial x} \frac{\partial^{2} \psi}{\partial x \partial y}+\frac{\delta}{(1+\delta x)^{2}} \times\left[3 \delta \frac{\partial^{2} \psi}{\partial x^{2}}-\frac{3 \delta^{2}}{1+\delta x} \frac{\partial \psi}{\partial x}\right] \\
& -\frac{2 \delta}{1+\delta x} \frac{\partial}{\partial x} \Delta_{2} \psi+w \frac{\partial w}{\partial y}+\Delta_{2}^{2} \psi-G r(1+\delta x) \frac{\partial T}{\partial x} \\
\frac{\partial T}{\partial t}+\frac{1}{(1+\delta x)} \frac{\partial(T, \psi)}{\partial(x, y)}= & \frac{1}{\operatorname{Pr}}\left(\Delta_{2} T+\frac{\delta}{1+\delta x} \frac{\partial T}{\partial x}\right)
\end{aligned}
$$

where,

$$
\Delta_{2} \equiv \frac{\partial^{2}}{\partial x^{2}}+\frac{\partial^{2}}{\partial y^{2}}, \frac{\partial(f, g)}{\partial(x, y)} \equiv \frac{\partial f}{\partial x} \frac{\partial g}{\partial y}-\frac{\partial f}{\partial y} \frac{\partial g}{\partial x} .
$$

The Dean number Dn, the Grashof number $G r$, and the Prandtl number Pr, which appear in Equations (2) to (4) are defined as

$$
D n=\frac{G d^{3}}{\mu v} \sqrt{\frac{2 d}{L}}, G r=\frac{\beta g \Delta T d^{3}}{v^{2}}, \operatorname{Pr}=\frac{v}{\kappa}
$$

The rigid boundary conditions for $w$ and $\psi$ are used as

$$
w( \pm 1, y)=w(x, \pm 1)=\psi( \pm 1, y)=\psi(x, \pm 1)=\frac{\partial \psi}{\partial x}( \pm 1, y)=\frac{\partial \psi}{\partial y}(x, \pm 1)=0
$$

and the temperature $T$ is assumed to be constant on the walls as

$$
T(1, y)=1, T(-1, y)=-1, T(x, \pm 1)=x .
$$

In the present study, $D n$ and $G r$ vary while $\operatorname{Pr}$ and $\delta$ are fixed as $\operatorname{Pr}=7.0$ (water) and curvature $\delta=0.1$.

\section{Numerical Calculations}

\subsection{Method of Numerical Calculation}

In order to solve the Equations (2) to (4) numerically the spectral method is used. This is the method which is thought to be the best numerical method to solve the Navier-Stokes equations as well as the energy equation (Gottlieb and Orazag, [21]). By this method the variables are expanded in a series of functions consisting of the Chebyshev polynomials. That is, the expansion functions

$$
\phi_{n}(x)=\left(1-x^{2}\right) C_{n}(x), \quad \psi_{n}(x)=\left(1-x^{2}\right)^{2} C_{n}(x)
$$

where $C_{n}(x)=\cos \left(n \cos ^{-1}(x)\right)$ is the nth order Chebyshev polynomial. $w(x, y, t), \psi(x, y, t)$ and $T(x, y, t)$ are expanded in terms of $\phi_{n}(x)$ and $\psi_{n}(x)$ as

$$
\left.\begin{array}{l}
w(x, y, t)=\sum_{m=0}^{M} \sum_{n=0}^{N} w_{m n}(t) \phi_{m}(x) \phi_{n}(y), \\
\psi(x, y, t)=\sum_{m=0}^{M} \sum_{n=0}^{N} \psi_{m n}(t) \psi_{m}(x) \psi_{n}(y), \\
T(x, y, t)=\sum_{m=0}^{M} \sum_{n=0}^{N} T_{m n}(t) \phi_{m}(x) \phi_{n}(y)+x,
\end{array}\right\}
$$

where $M$ and $N$ are the truncation numbers in the $x$ and $y$ directions respectively. In this study, for necessary accuracy of the solutions, we use $M=20$ and $N=20$. In order to calculate the unsteady solutions, the Crank-Nicolson and Adams-Bashforth methods together with the function expansion (10) and the collocation methods are applied. 


\subsection{Resistance Coefficient}

The resistant coefficient $\lambda$ is used as the representative quantity of the flow state. It is also called the hydraulic resistance coefficient, and is generally used in fluids engineering, defined as

$$
\frac{P_{1}^{*}-P_{2}^{*}}{\Delta z^{*}}=\frac{\lambda}{d_{h}^{*}} \frac{1}{2} \rho\left\langle w^{*}\right\rangle^{2},
$$

where, quantities with an asterisk $\left(^{*}\right)$ denote dimensional ones, \langle\rangle stands for the mean over the cross section of the duct and $d_{h}^{*}$ is the hydraulic diameter. The main axial velocity $\left\langle w^{*}\right\rangle$ is calculated by

$$
\left\langle w^{*}\right\rangle=\frac{v}{4 \sqrt{2 \delta} d} \int_{-1}^{1} \mathrm{~d} x \int_{-1}^{1} \bar{w}(x, y, t) \mathrm{d} y .
$$

Since $\left(P_{1}^{*}-P_{2}^{*}\right) / \Delta_{z^{*}}=G, \lambda$ is related to the mean non-dimensional axial velocity $\langle w\rangle$ as

$$
\lambda=\frac{4 \sqrt{2 \delta} D n}{\langle w\rangle^{2}},
$$

where, $\langle w\rangle=\sqrt{2 \delta} d\left\langle w^{*}\right\rangle / v$. In the present study, $\lambda$ is used to perform time evolution of the unsteady solutions.

\section{Results and Discussion}

\subsection{Time Evolution of the Unsteady Solutions}

Time evolution of the resistance coefficient $\lambda$ are performed for $D n=100$ and $100 \leq G r \leq 2000$ as shown in Figure 2(a). It is found that the flow is a steady-state solution for $D n=100$ and $100 \leq G r \leq 2000$. To draw

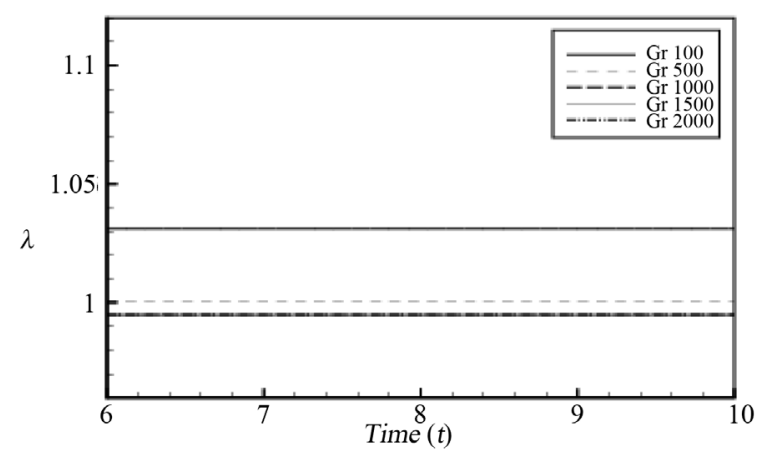

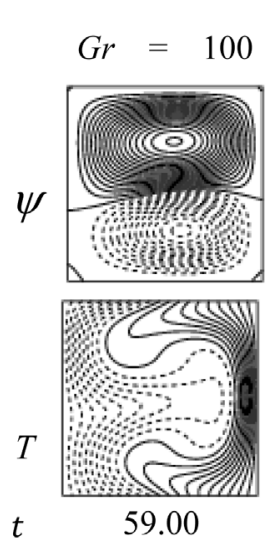

$t$
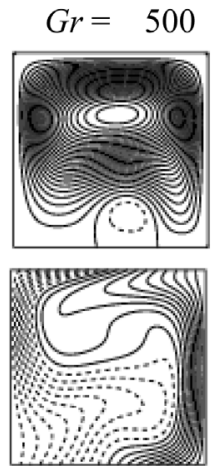

84.20

(a)
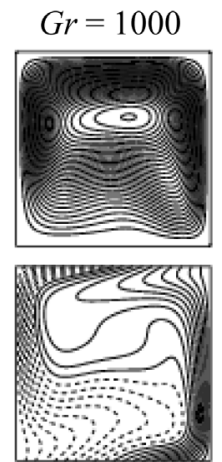

85.00
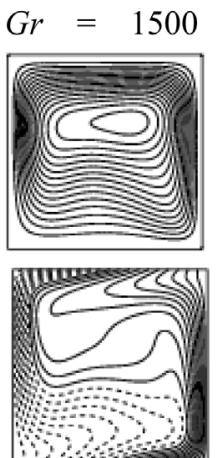

25.00
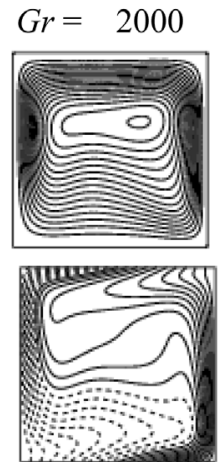

85.00

(b)

Figure 2. (a) Time-dependent flow for $D n=100$ and $100 \leq G r \leq 2000$; (b) Secondary flow patterns (top) and temperature profiles (bottom) for $D n=100$ and $100 \leq G r \leq 2000$. 
the contours for the stream lines of the secondary flow patterns $(\psi)$ and temperature profiles $(T)$, we use the increments $\Delta \psi=0.6$ and $\Delta T=0.25$ respectively. The same increments of $\psi$ and $T$ are used for all the figures in this study, unless specified. The right-hand side of each duct box of $\psi$ and $T$ is in the outside direction of the duct curvature. In the figures of the stream lines, solid lines $(\psi \geq 0)$ show that the secondary flow is in the counter clockwise direction while the dotted lines $(\psi<0)$ in the clockwise direction. Similarly, in the figures of the isotherms (temperature profiles), solid lines are those for $T \geq 0$ and dotted ones for $T<0$. Since the flow is steady-state, single contours of the secondary flow patterns and temperature profiles are shown in Figure 2(b), where it is seen that the unsteady flow is an asymmetric single- and two-vortex solution. It is found that as $G r$ increases, the two-vortex solution ceases to be a single-vortex solution which covers the whole cross-section of the duct. We also investigated time-dependent solutions for $D n=500$ and $100 \leq G r \leq 2000$ and obtained same type of flow behavior as obtained for $D n=100$. The results are shown in Figure 3, where we find that the unsteady flow is an asymmetric two-vortex steady-state solution.

Then, we investigated time-dependent solutions of $\lambda$ for $D n=1000$ and $100 \leq G r \leq 2000$. The results are shown in Figure 4(a). As seen in Figure 4(a), the time-dependent flow is a periodic solution for $G r=100$ and $G r=500$ but steady-state solution for $1000 \leq G r \leq 2000$. In order to see the flow oscillations more clearly, we explicitly show time variations of $\lambda$ for $G r=100$ in Figure 4(b), where periodic flows are clearly observed. Contours of secondary flow patterns and temperature profiles are shown in Figure 4(c) for $23.45 \leq t \leq 25.40$. It is found that the periodic flow for $G r=100$ oscillates between asymmetric four-vortex solution. Figure 5(a) explicitly shows time evolution of $\lambda$ for $D n=1000$ and $G r=500$, where it is seen that the flow oscillates periodically. Corresponding secondary flow patterns and temperature profiles are shown in Figure 5(b) for $42.08 \leq t \leq 42.30$. As seen in Figure 5(b), the periodic oscillation for $G r=500$ oscillates between asymmetric two-vortex solutions. Since the unsteady flow is a steady-state solution for $100 \leq G r \leq 2000$, typical contours of the secondary flow patterns and temperature profiles are shown in Figure 5(c) for $G r=1000,1500$ and 2000. In Figure 5(c), we see that the steady-state flow for $D n=1000$ and $G r=1000,1500$ and 2000 are

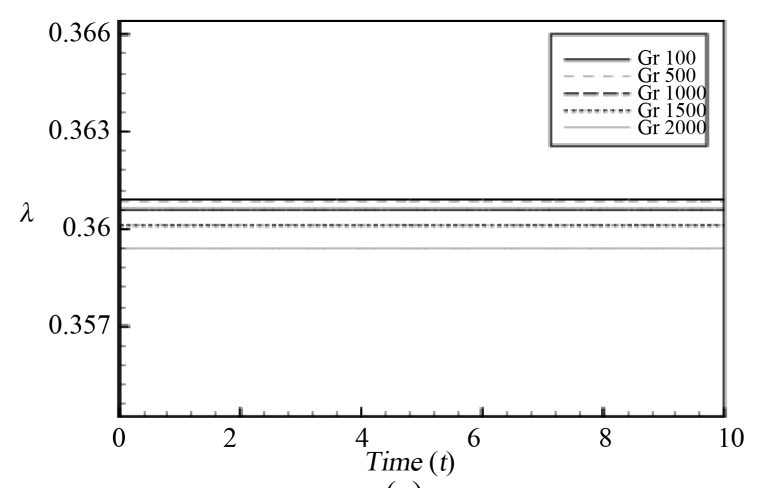

(a)

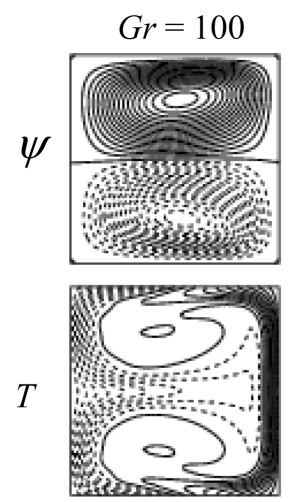

28.00
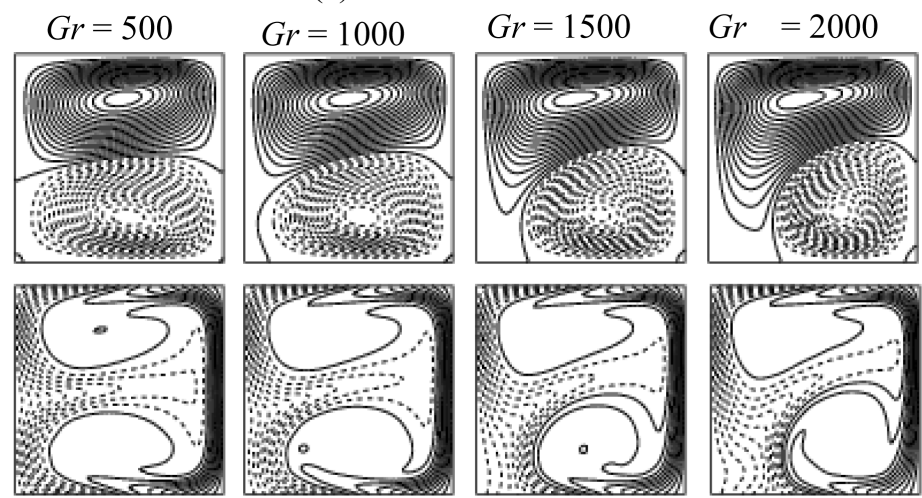

32.00

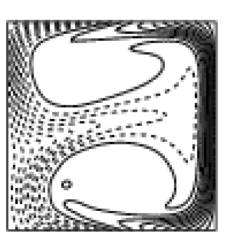

88.00

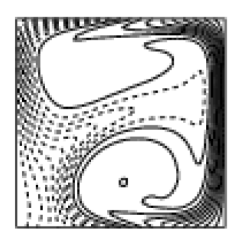

89.50

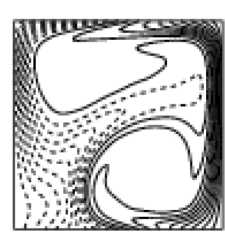

87.00

(b)

Figure 3. (a) Time-dependent flow for $D n=500$ and $100 \leq G r \leq 2000$; (b) Secondary flow patterns (top) and temperature profiles (bottom) for $D n=500$ and $100 \leq G r \leq 2000$. 


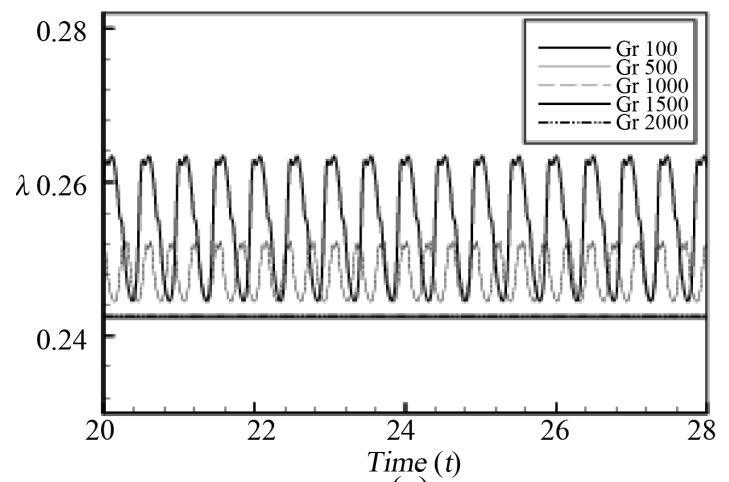

(a)

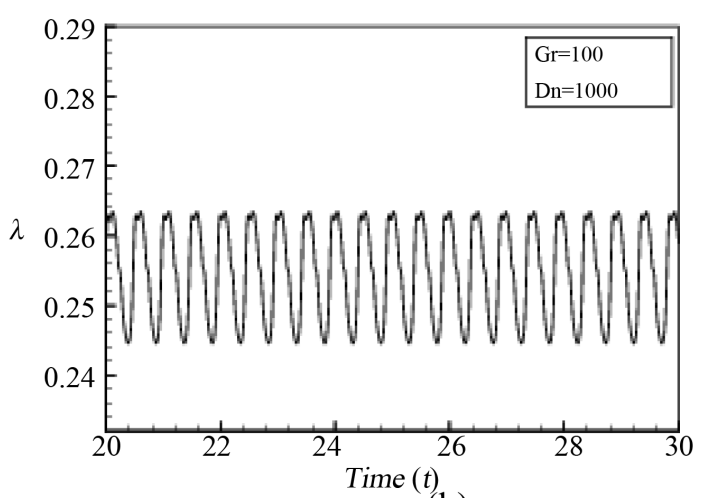

(b)

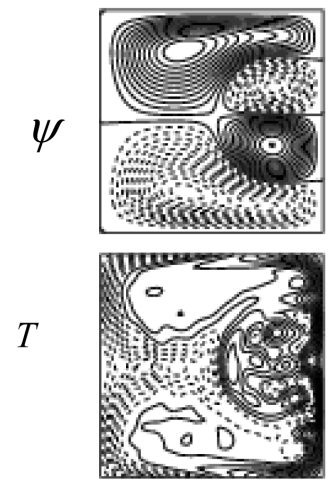

23.45

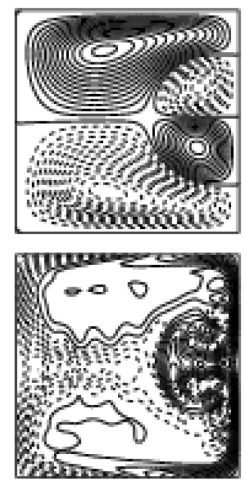

23.90

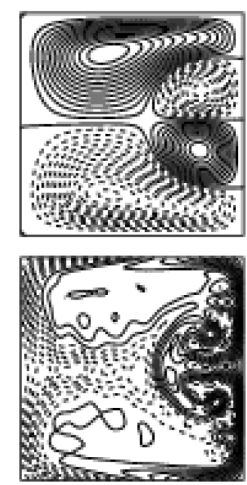

24.40

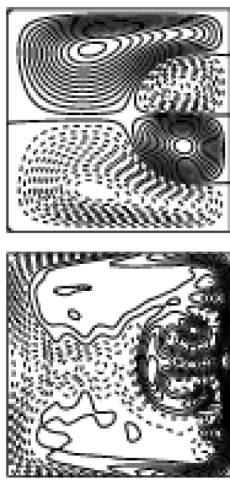

24.90
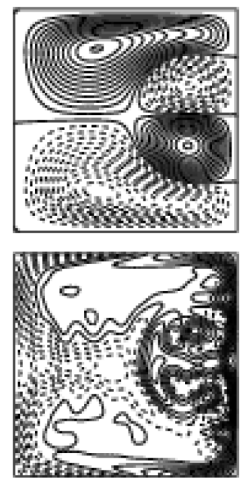

25.40

(c)

Figure 4. (a) Time-dependent flow for $D n=1000$ and $100 \leq G r \leq 2000$; (b) time evolution of $\lambda$ for $D n=1000$ and $G r=100$; (c) secondary flow patterns (top) and temperature profiles (bottom) for $D n=1000$ and $G r=100$.

asymmetric two-vortex solution.

We then performed time evolution of $\lambda$ for $D n=1500$ and $100 \leq G r \leq 2000$. The result is shown in Figure 6(a). As seen in Figure 6(a), the time-dependent flow for $D n=1500$ is a steady-state solution for all $100 \leq G r \leq 2000$. Secondary flow patterns and temperature profiles, depicted in Figure 6(b) for $D n=1500$, shows that the flow is an asymmetric two-vortex solution. The temperature profile is consistent with the secondary vortices. The result of the time-dependent solution of $\lambda$ for $D n=2000$ and $100 \leq G r \leq 2000$ is shown in Figure 7(a). As seen in Figure 7(a), the time-dependent flow for $D n=2000$ is a steady-state solution for $100 \leq G r \leq 1500$ but periodic oscillating flow for $G r=2000$. The time-periodic flow for $G r=2000$ is individually shown in Figure 7(b) for a clear view. Figure 7(c) shows typical contours of secondary flow patterns and temperature profiles for the steady-state solutions at $100 \leq G r \leq 1500$ and Figure 7(d) shows those for $G r=2000$, for one period of oscillation at time $20.68 \leq t \leq 21.01$, and we find that both the time-periodic and steady-state solutions are asymmetric two-vortex solutions. Then we show the results of the time-dependent solutions for $\mathrm{Dn}=2500$ and $100 \leq G r \leq 2000$ in Figure 8(a). As seen in Figure 8(a), the time-dependent flow for $D n=2500$ is a steady-state solution for $100 \leq G r \leq 1000$ but periodic oscillating flow for $G r=1500$ and multi-periodic (or transitional chaos) flow for $G r=2000$. Figures 8(b)-(d) respectively show those of the time-dependent solutions for the steady-state solutions at $G r=100,500$ and 1000. Since the flow is steady-state at $G r=100,500$ and 1000, a single contour of each of the secondary flow patterns and temperature profiles is shown in Figures 8(b)-(d) respectively. Figure 9(a) and Figure 9(b) respectively show time-dependent solutions for $G r=1500$ and 2000 at $D n=2500$. As seen in Figure 9(a) and Figure 9(b), the unsteady flow is a time-periodic for $G r=1500$ but multi-periodic (sometimes called transitional chaos, (Mondal et al. [14]) for $G r=2000$. Typical contours of secondary flow patterns and temperature distributions for $G r=1500$ and 2000 are shown in Figure 9(c) and Figure 9(d) respectively, where we see that the periodic or multi-periodic 


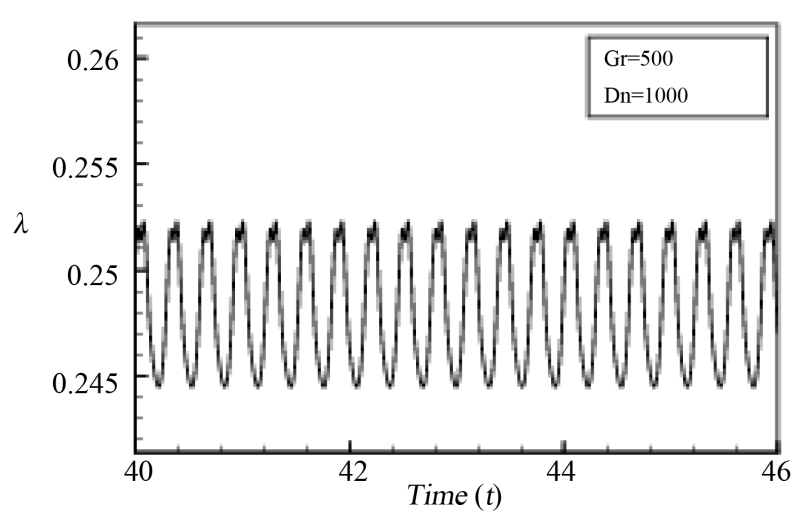

(a)
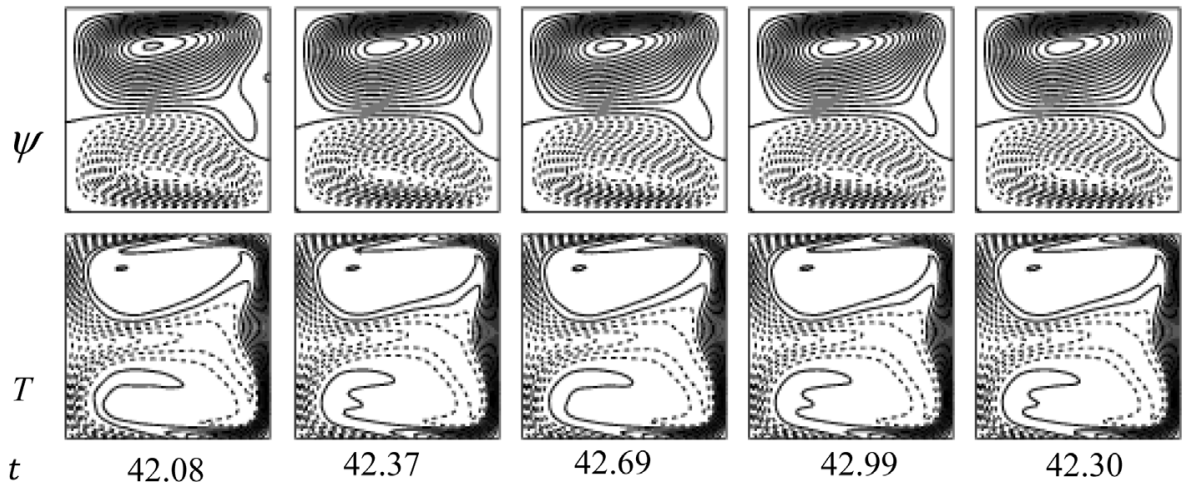

42.69

42.99

42.30

(b)
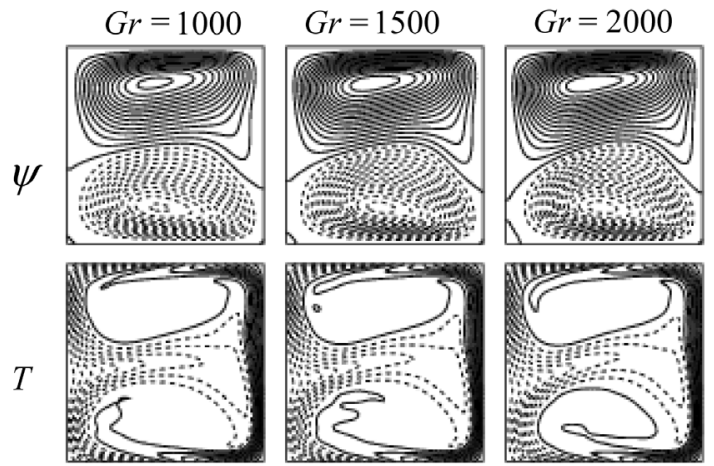

$t$

68.30

24.92

24.93

(c)

Figure 5. (a) Time-dependent flow for $D n=1000$ and $G r=500$ at time $40 \leq t \leq 46$; (b) secondary flow patterns (top) and temperature profiles (bottom); (c) single contours of secondary flow patterns (top) and temperature profiles (bottom) for $G r=1000,1500$ and 2000 at $D n=1000$.

flows are asymmetric two-vortex solution.

Finally, the results of the time-dependent solutions for $D n=3000$ and $100 \leq G r \leq 2000$ are unitedly shown in Figure 10(a) and separately in the successive figures. Figure 10(b) explicitly show time-dependent flow for $D n=3000$ and $G r=100$ and it is found that the flow is multi-periodic rather than periodic. Then, in order to see the multi-periodic oscillation more clearly, a phase space of the time change of $\lambda$ for $\mathrm{s}$ and $G r=100$ is shown in Figure 10(c) in the $\lambda-\gamma$ plane, where $\gamma=\iint \psi \mathrm{d} x \mathrm{~d} y$. As seen in Figure 10(c), the time-dependent flow creates multiple orbits, which suggests that the flow is multi-periodic. Typical contours 


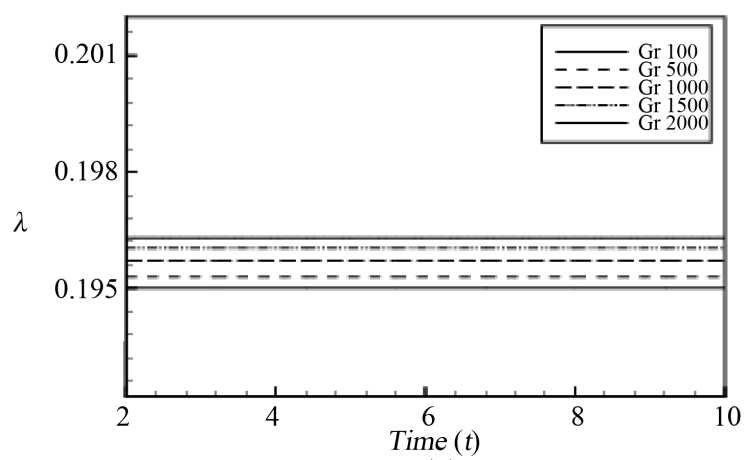

(a)
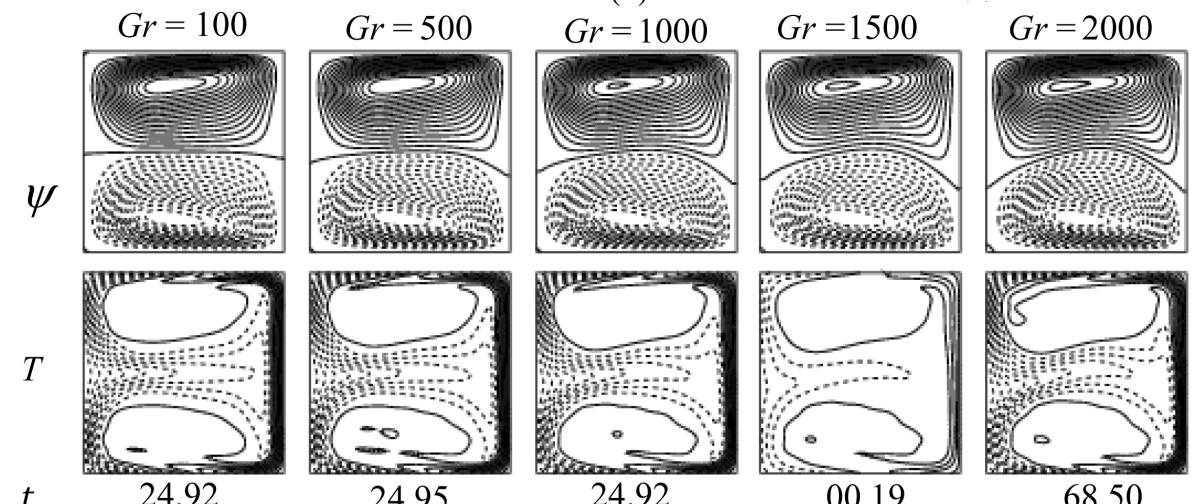

24.92

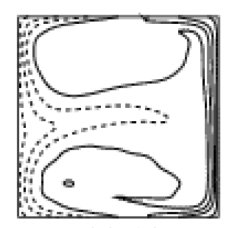

00.19

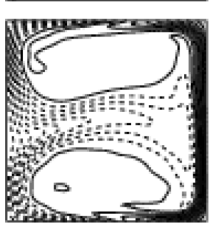

68.50

(b)

Figure 6. (a) Time-dependent flow for $D n=1500$ and $100 \leq G r \leq 2000$; (b) contours of secondary flow patterns (top) and temperature profiles (bottom) for $D n=1500$ and $100 \leq G r \leq 2000$.

of secondary flow patterns and temperature profiles are shown in Figure 10(d), and it is found that the flow oscillates between asymmetric two-vortex solutions. Then we explicitly show the result of the time-dependent flow for $D n=3000$ and $G r=500$ in Figure 11(a). Then, to be sure whether the flow is periodic, multi-periodic or chaotic, we draw the phase space of the time-dependent flow for $D n=3000$ and $G r=500$ in Figure 11(b) and see that the flow is a transitional chaos (Mondal [7]). Then we draw typical contours of secondary flow patterns and temperature profiles for the transitional chaos at $D n=3000$ and $G r=500$ in Figure 11(c). Figure 11(c) shows that the flow is an asymmetric two-vortex solution. Then we perform time-evolution of $\lambda$ for $D n=3000$ and $G r=1000$, and presented in Figure 12(a). As seen in Figure 12(a), the flow oscillates multi-periodically. In order to see the characteristics of the multi-periodic oscillation, we draw the phase space of the time-dependent flow for $D n=3000$ and $G r=1000$ and presented in Figure 12(b). It is found that the unsteady flow creates irregular or multiple orbit which means the flow presented in Figure 12(a) is chaotic rather than multi-periodic. The chaotic behavior is clearly justified by Figure 12(b). Then we draw typical contours of secondary flow patterns and temperature profiles for the chaotic oscillation at $D n=3000$ and $G r=1000$ in Figure 12(c). As seen in Figure 12(c), the chaotic flow oscillates irregularly between the asymmetric two-vortex solutions. The results of the time-dependent flow for $G r=1500$ and $G r=2000$ at $D n=3000$ are shown in Figure 13(a) and Figure 14(a) respectively. As seen in Figure 13(a) and Figure 14(a), the unsteady flows at $G r=1500$ and $G r=2000$ are periodic solutions, which are well justified by drawing the phase-spaces as shown in Figure 13(b) and Figure 14(b) respectively. It is found that the two flows have nearly the same type of unsteady flow behavior. Typical contours of secondary flow patterns and temperature profiles for the periodic oscillations at $G r=1500$ and $G r=2000$ for $D n=3000$ are shown in Figure 13(c) and Figure 14(c) respectively, where we see that the periodic flow oscillates between asymmetric two-vortex solutions. The temperature distribution is well consistent with the secondary vortices, and it becomes so entan- 
gled when the secondary vortices become stronger. In this regard, it should be worth mentioning that irregular oscillation of the non-isothermal and isothermal flows has been observed experimentally by Wang and Yang [10] for a curved square duct flow and by Ligrani and Niver [22] for flow through a curved rectangular duct of large aspect ratio.

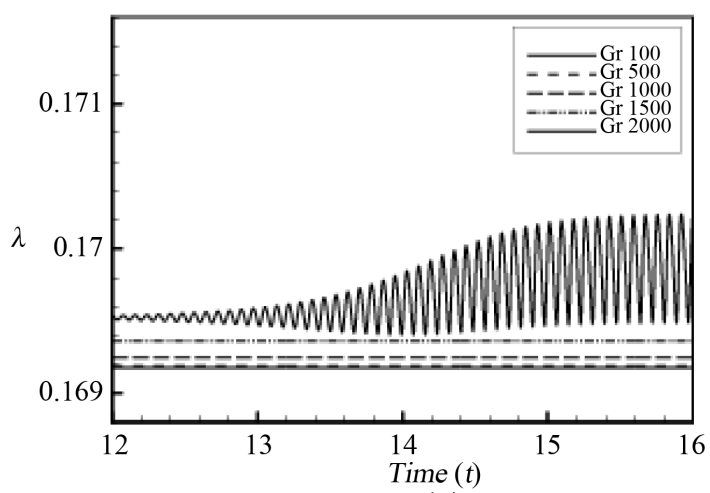

(a)

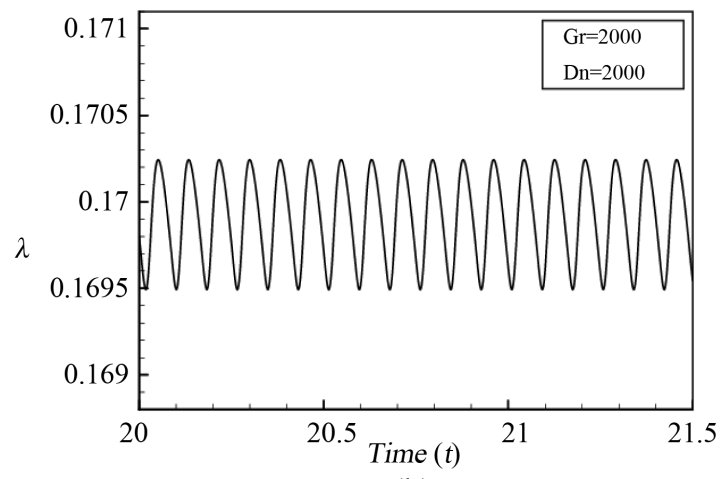

(b)
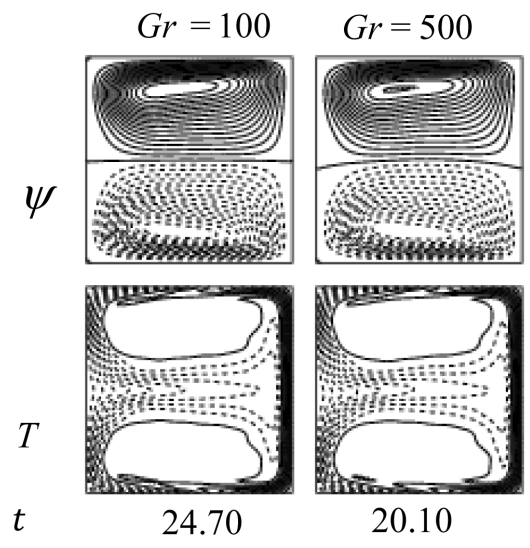

$G r=1000$
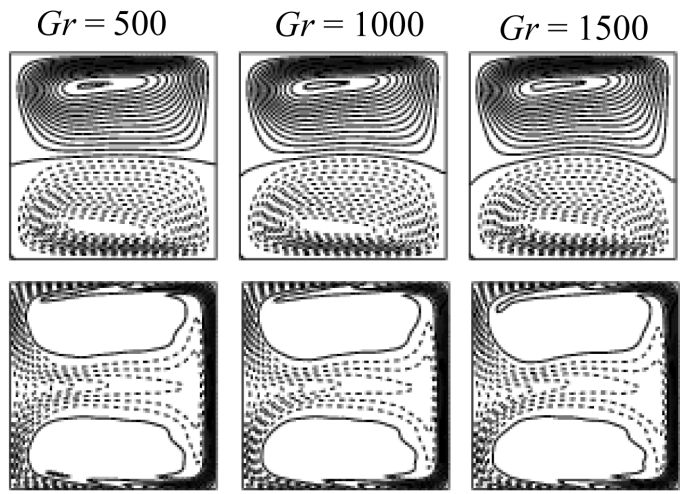

20.10

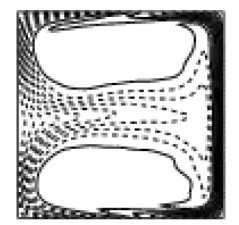

24.30

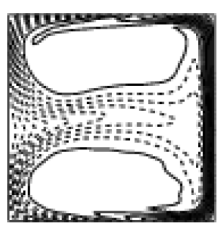

24.20

(c)
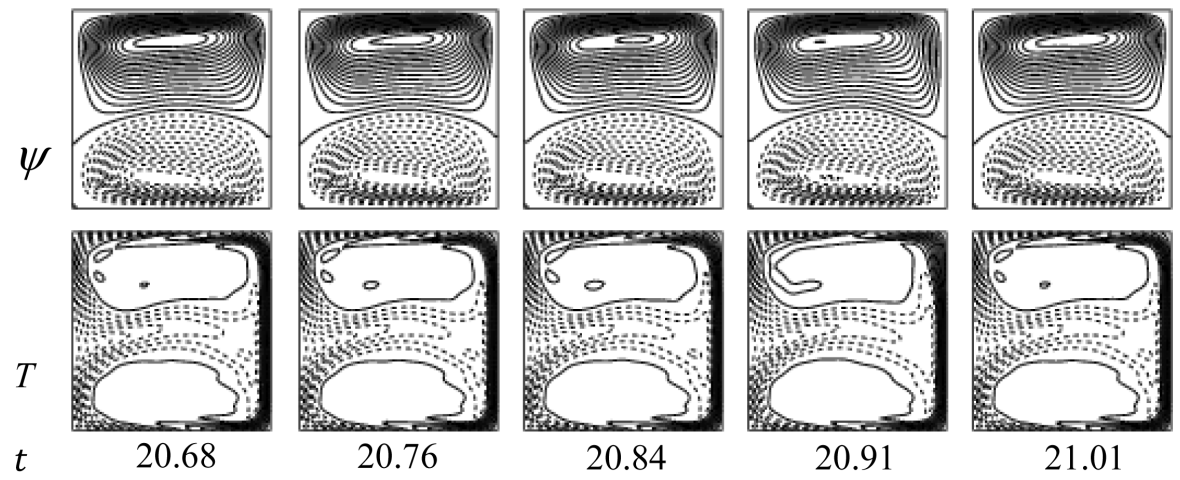

20.84

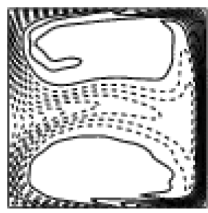

20.91

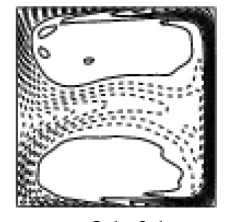

(d)

Figure 7. (a) Time-dependent flow for $D n=2000$ and $100 \leq G r \leq 2000$; (b) time evolution of $\lambda$ for $G r=2000$; (c) flow patterns for $100 \leq G r \leq 1500$; (d) contours of secondary flow patterns and temperature profiles for $G r=2000$. 


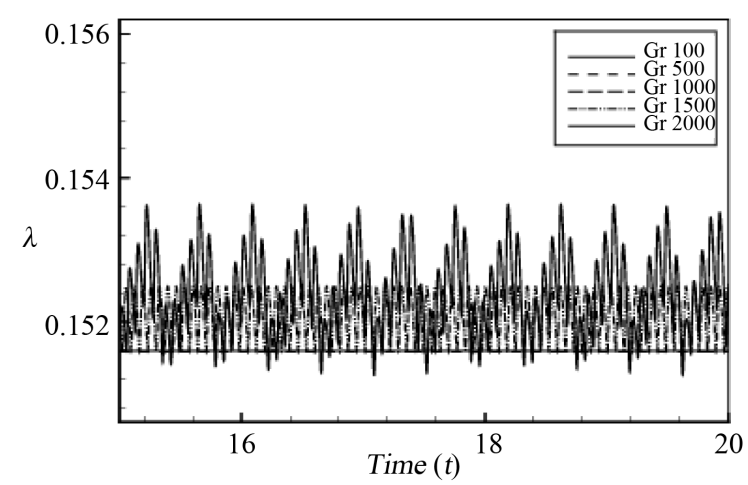

(a)

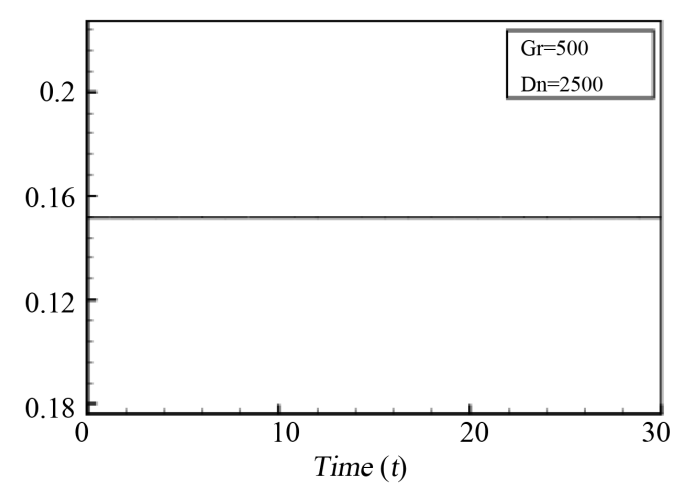

(c)

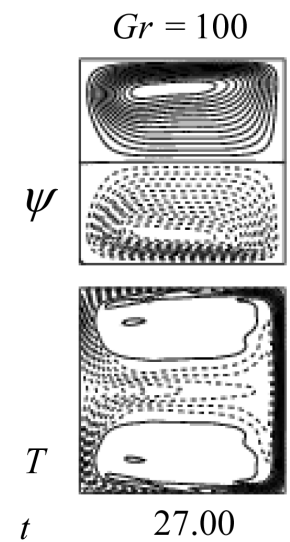

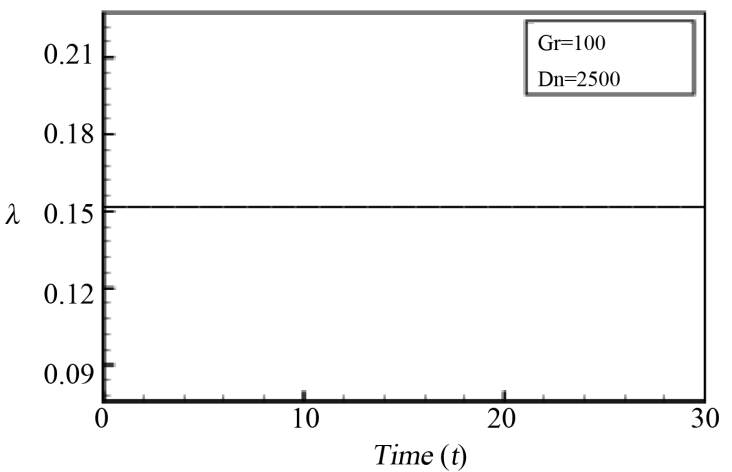

(b)

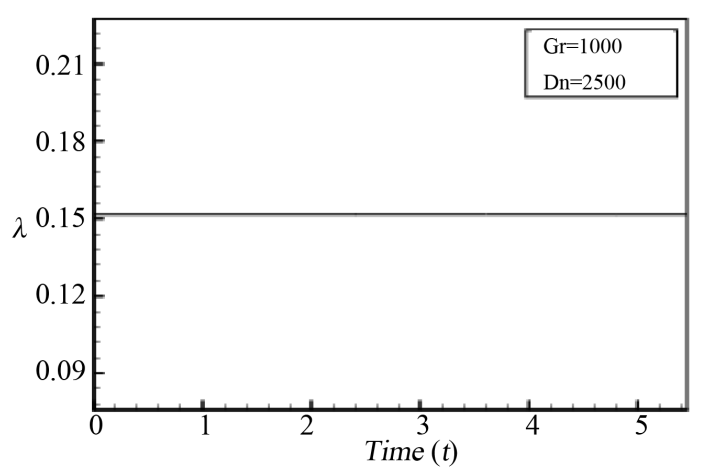

(d)

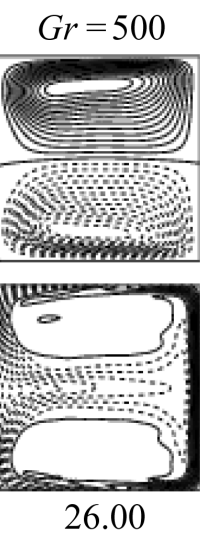

(e)
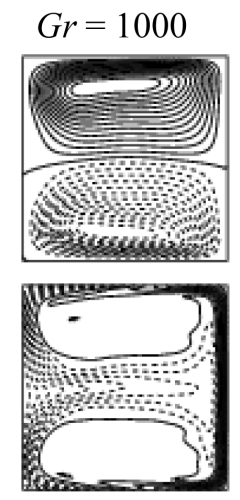

05.40

Figure 8. (a) Time-dependent flow for $D n=2500$ and $100 \leq G r \leq 2000$; (b) time evolution of $\lambda$ for $G r=100$; (c) time evolution of $\lambda$ for $G r=500$; (d) time evolution of $\lambda$ for $G r=1000$; (e) contours of secondary flow patterns and temperature profiles for $G r=100,500$ and 1000 at $D n=2500$. 


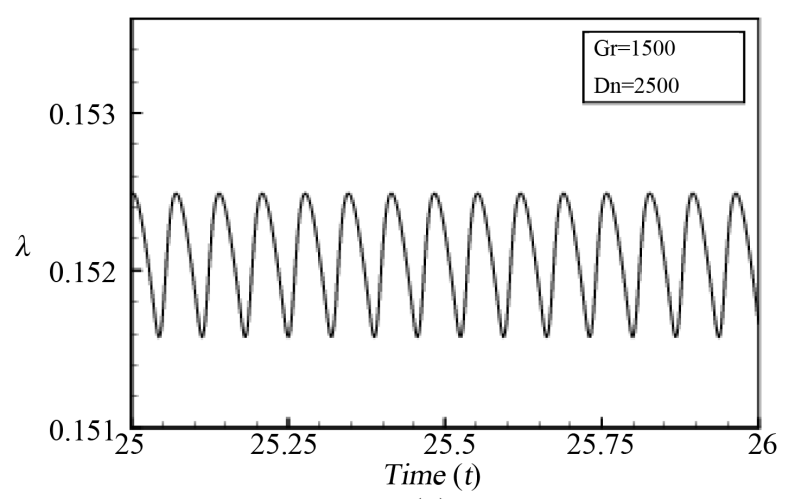

(a)

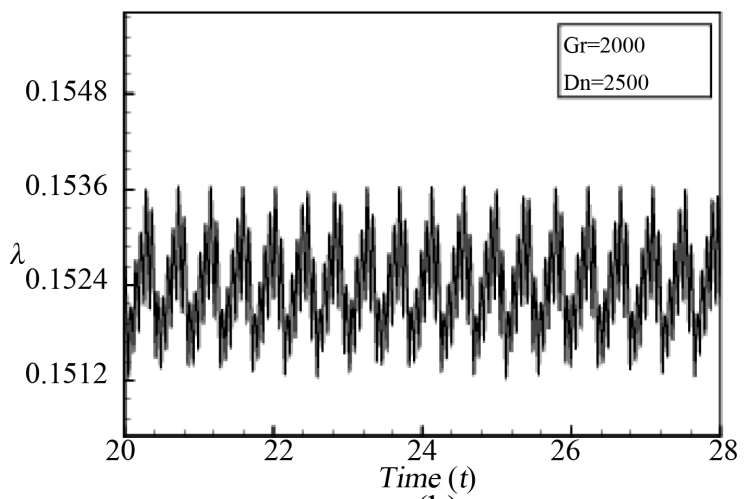

(b)
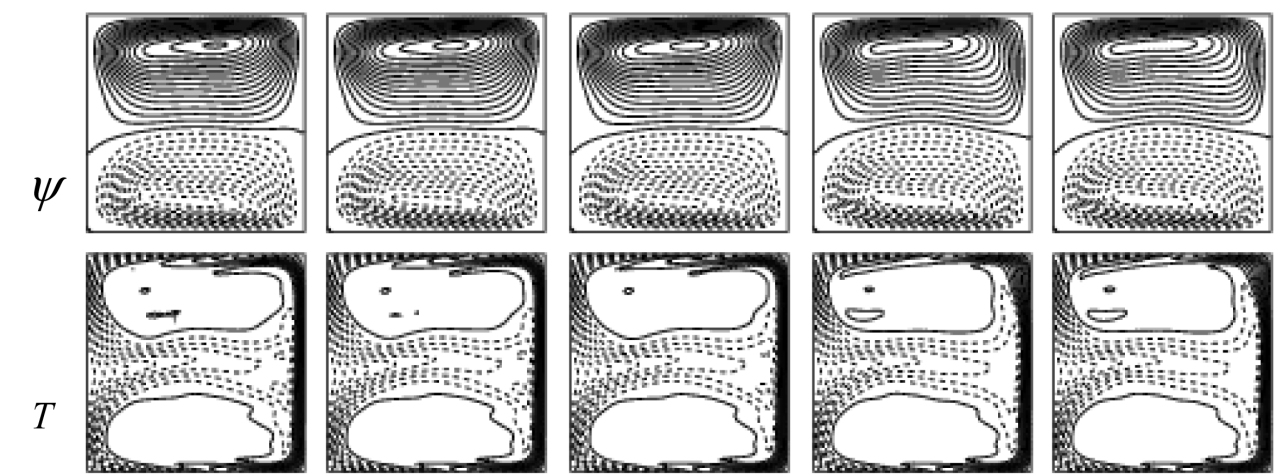

25.25

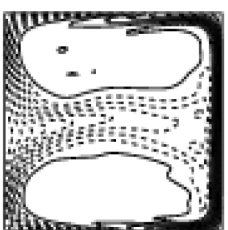

25.32

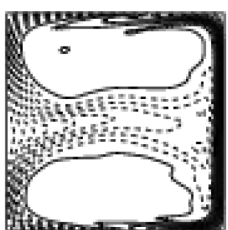

25.39

(c)
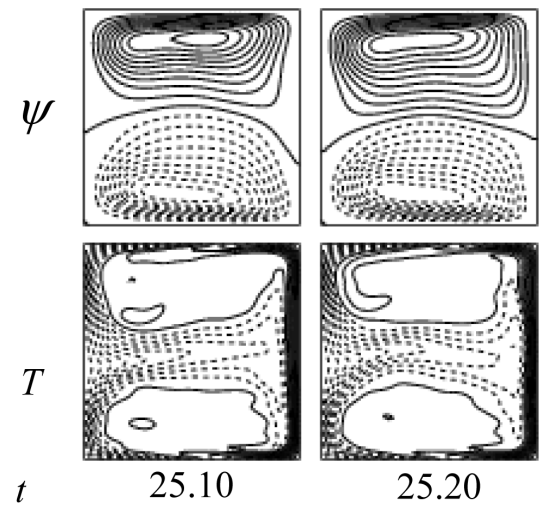

25.20

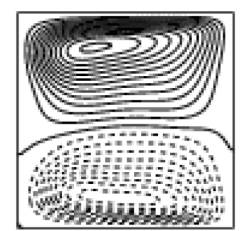

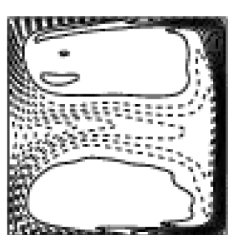

25.51

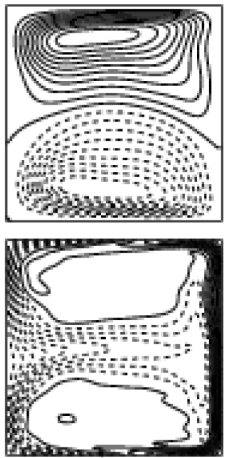

25.40

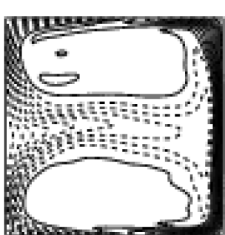

25.58
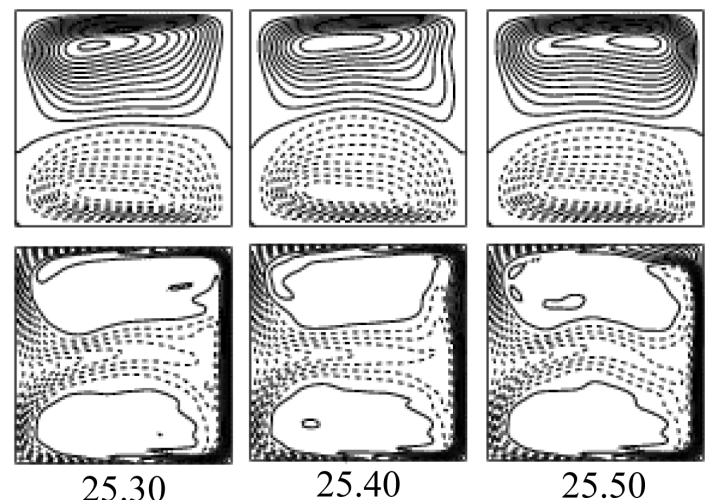

(d)

Figure 9. (a) Time-dependent flow for $D n=2500$ and $G r=1500$; (b) time evolution of $\lambda$ for $D n=2500$ and $G r=2000$; (c) contours of secondary flow patterns (top) and temperature profiles (bottom) for $G r=1500$; (d) contours of secondary flow patterns (top) and temperature profiles (bottom) for $G r=2000$. 


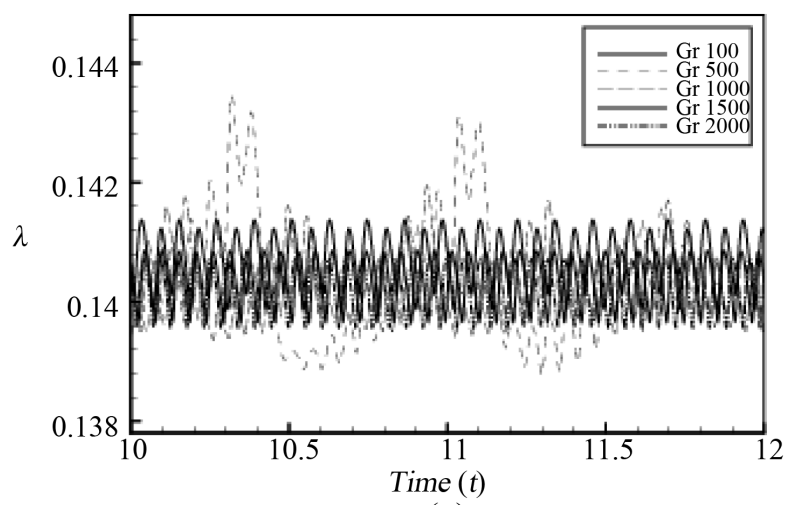

(a)

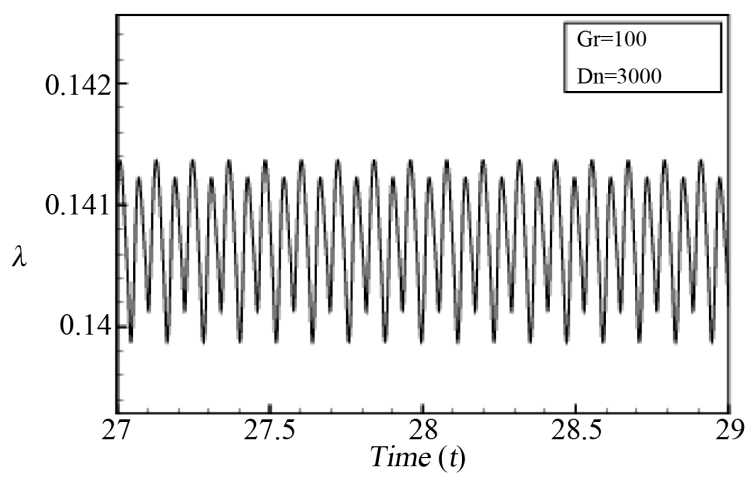

(b)

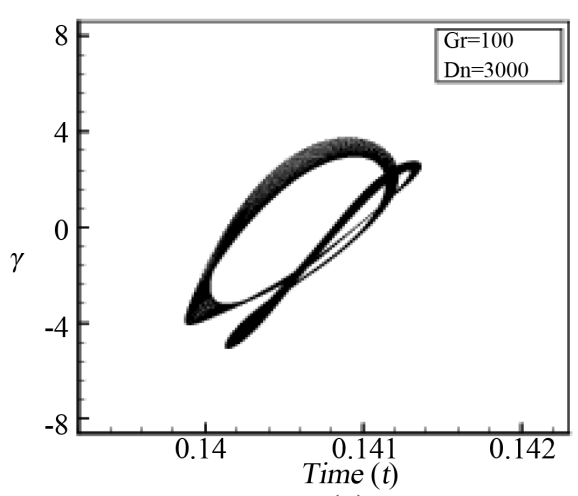

(c)
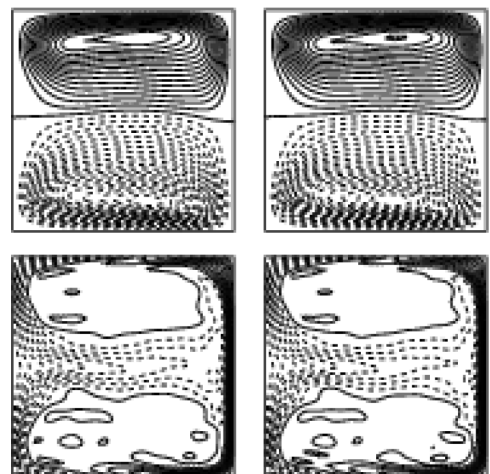

28.35

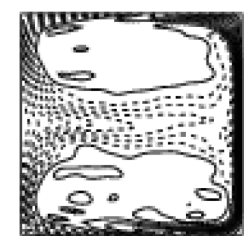

28.47

(d)

Figure 10. (a) Time-dependent flow for $D n=3000$ and $100 \leq G r \leq 2000$; (b) time evolution of $\lambda$ for $D n=3000$ and $G r=100$; (c) phase space; (d) secondary flow patterns and temperature profiles for $D n=3000$ and $G r=100$. 


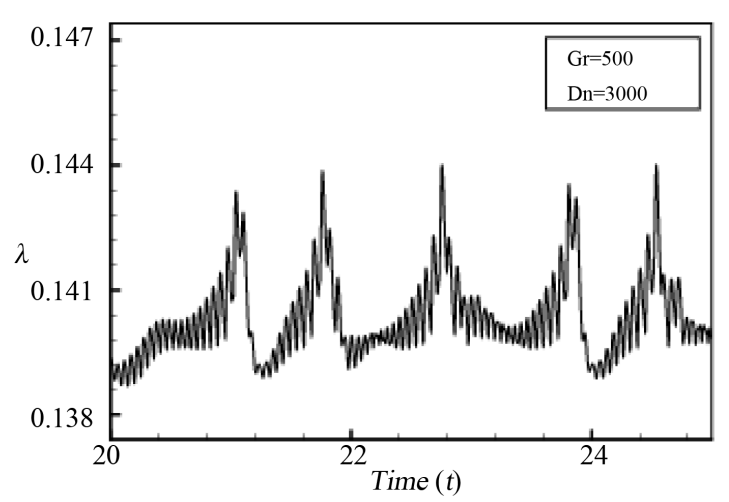

(a)

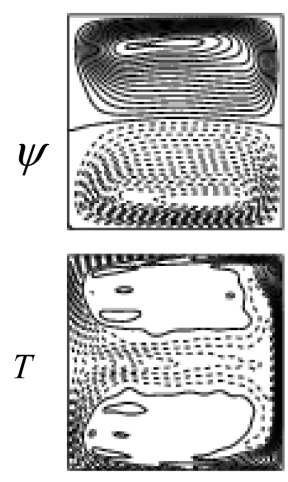

28.00
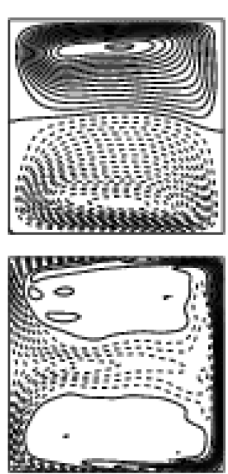

28.50

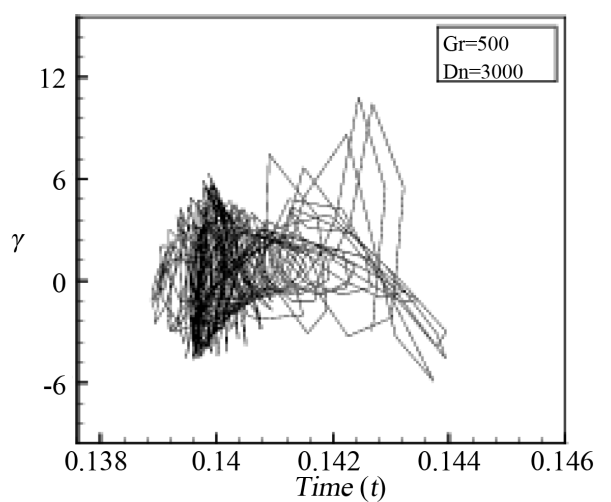

(b)

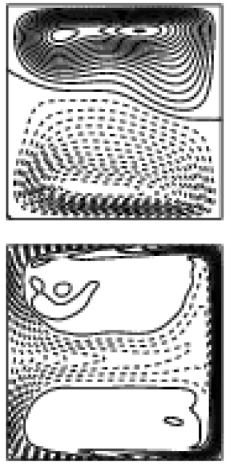

29.00
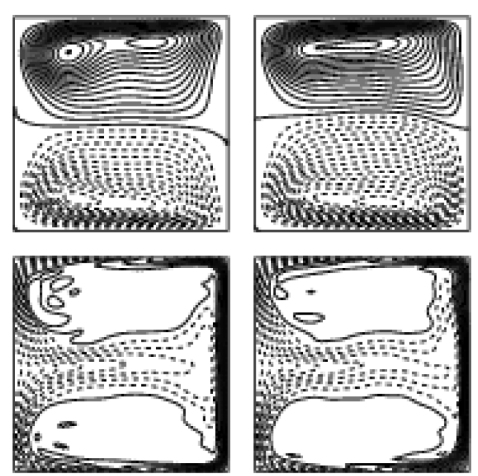

29.50

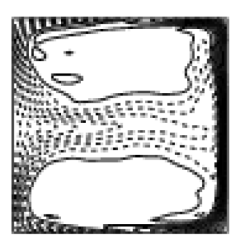

30.00

Figure 11. (a) Time-dependent flow for $D n=3000$ and $G r=500$; (b) phase space for $D n=3000$ and $G r=500$; (c) secondary flow patterns (top) and temperature profiles (bottom) for $D n=3000$ and $G r=500$.

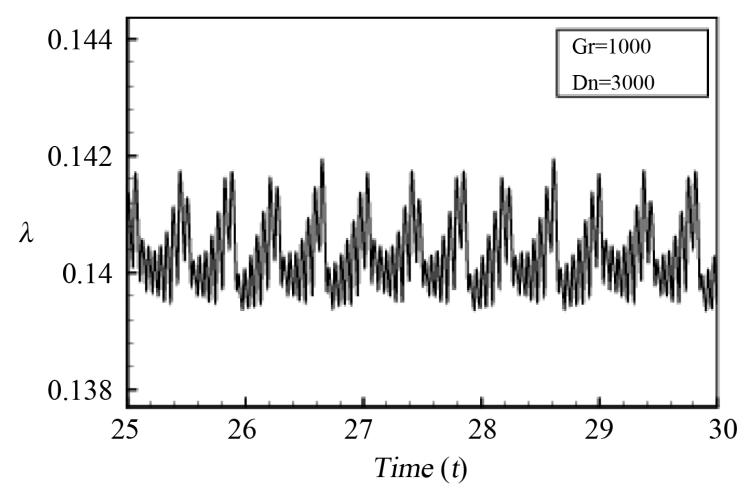

(a)

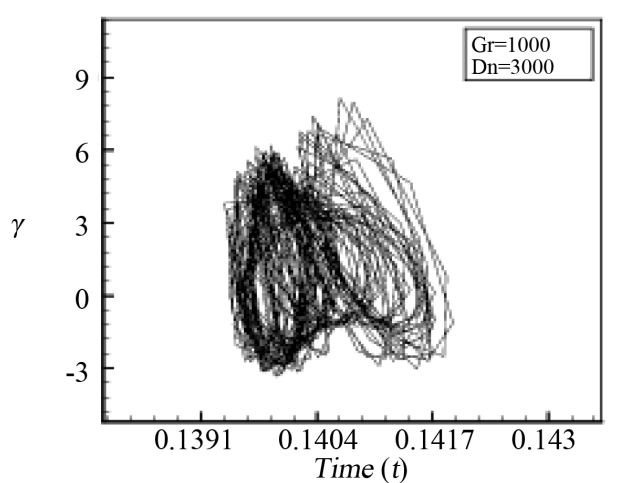

(b)

Figure 12. (a) Time-dependent flow for $D n=3000$ and $G r=1000$; (b) phase space for $D n=3000$ and $G r=1000$. 

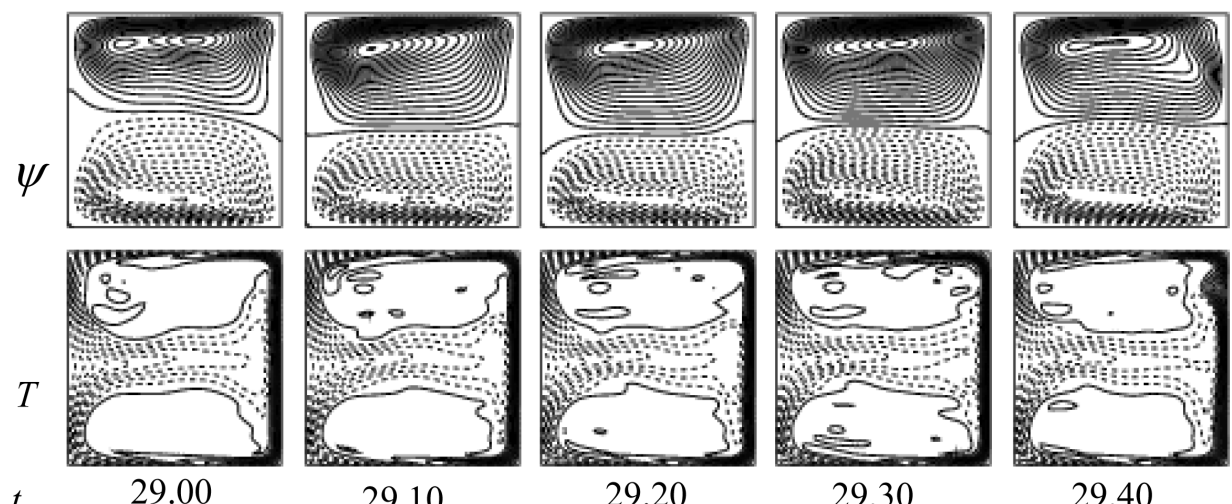

29.10

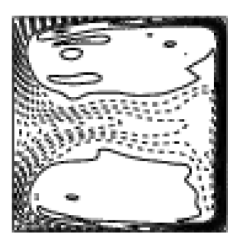

29.20

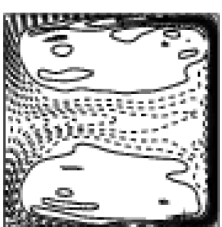

29.30

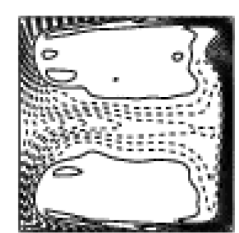

29.40

(c)

Figure 12. (c) Secondary flow patterns (top) and temperature profiles (bottom) for $G r=1000$.

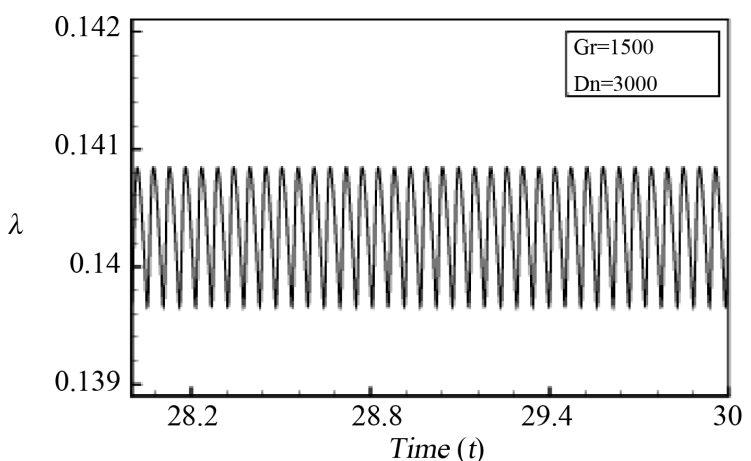

(a)
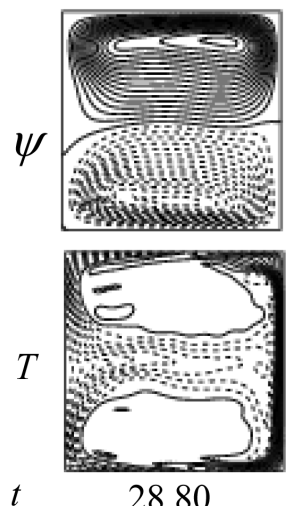

$t \quad 28.80$
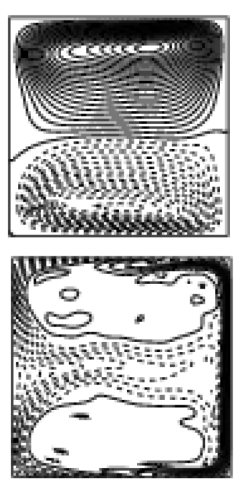

28.86
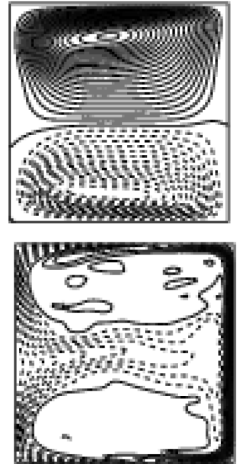

28.92

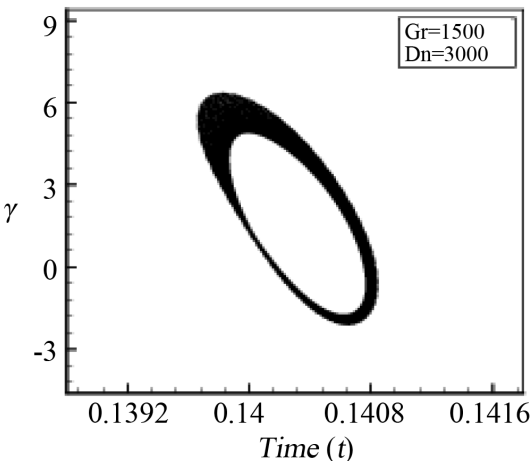

(b)
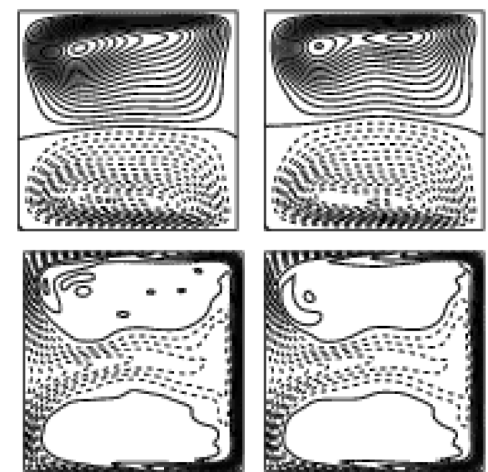

28.98

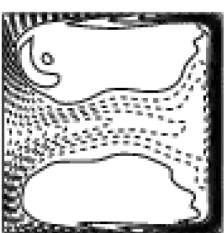

29.04

(c)

Figure 13. (a) Time-dependent flow for $D n=3000$ and $G r=1500$; (b) phase space for $D n=3000$ and $G r=1000$; (c) secondary flow patterns (top) and temperature profiles (bottom) for $D n=3000$ and $G r=1500$. 


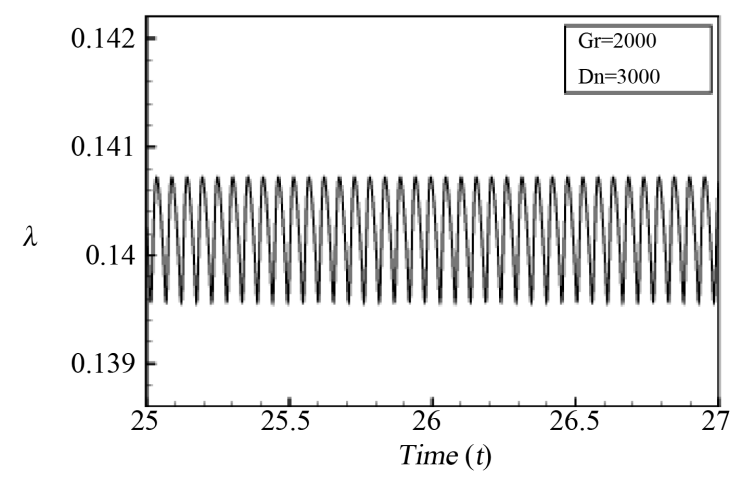

(a)
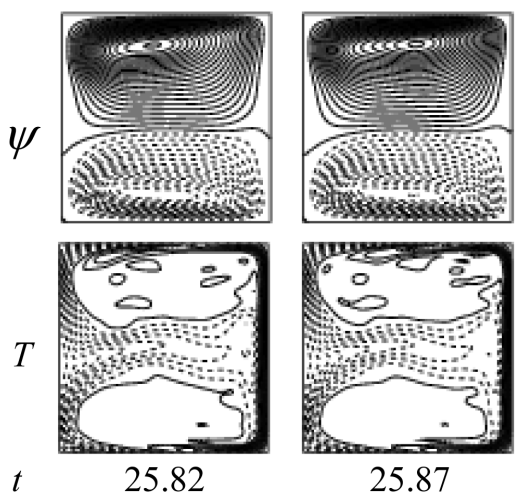

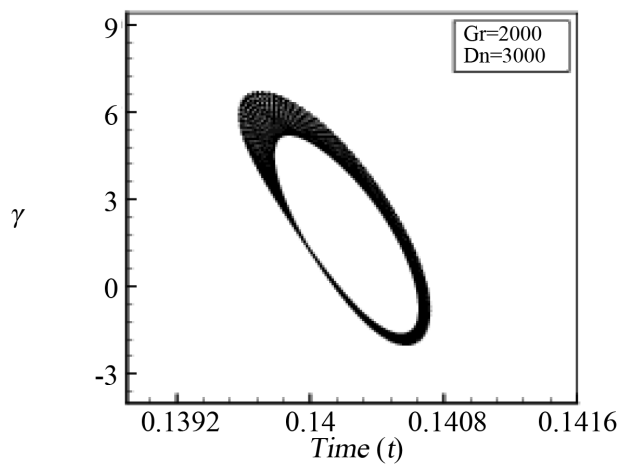

(b)

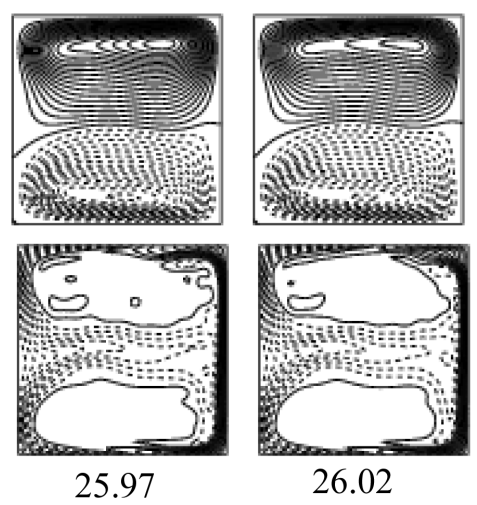

25.92

(c)

Figure 14. (a) Time-dependent flow for $D n=3000$ and $G r=2000$; (b) phase space for $D n=3000$ and $G r=2000$; (c) secondary flow patterns (top) and temperature profiles (bottom) for $D n=3000$ and $G r=2000$.

\subsection{Phase Diagram in the Dn-Gr Plane}

Finally, the distribution of the time-dependent solutions, obtained by the time evolution calculations of the curved square duct flows, is shown in Figure 15 in the Dean number versus Grashof number (Dn-Gr) plane for $100 \leq D n \leq 3000$ and $100 \leq G r \leq 2000$, where the circle indicates steady-state solutions, the cross periodic (or multi-periodic) solutions and the triangle chaotic solutions. As seen in Figure 15, the unsteady flow is always a steady-state solution for any value of $G r$ in the range $100 \leq G r \leq 2000$, when $100 \leq D n \leq 1500$ except for $D n=1000$. At $D n=1000$, the flow is periodic for $G r=100$ and 500 but steady-state otherwise. For $D n=$ 2000, the flow is periodic at $G r=2000$ but steady-state when $G r<2000$. As seen in Figure 15, the flow is also periodic/multi-periodic for $D n=2500$ at $G r=1500$ and 2000; for large Dean numbers, e.g. $D n=3000$, on the other hand, the unsteady flow changes in the scenario "periodic $\rightarrow$ chaotic $\rightarrow$ periodic", if Gr in increased.

\section{Conclusion}

A numerical study is presented for the time-dependent solutions of the flow through a curved square duct of constant curvature $\delta=0.1$. Numerical calculations are carried out by using a spectral method, and covering a wide range of the Dean number $100 \leq D n \leq 3000$ and the Grashof number $100 \leq G r \leq 2000$. A temperature difference is applied across the vertical sidewalls, where the outer wall is heated and the inner wall cooled. In order to study the non-linear behavior of the unsteady solutions, we performed time evolution calculations and it is found that the unsteady flow is a steady-state solution for $D n=100,500$ and 1500 for all values of the Gr investigated in this study. However, the unsteady flow is periodic for $D n=1000$ at $G r=100,500$ and for $D n=$ 2000 at $G r=2000$. The unsteady flow is also periodic/multi-periodic for $D n=2500$ at $G r=1500$ and 2000. For large Dean numbers, e.g. $D n=3000$, on the other hand, the unsteady flow undergoes in the scenario "periodic $\rightarrow$ chaotic $\rightarrow$ periodic", if $G r$ is increased. Typical contours of secondary flow patterns and temperature profiles 


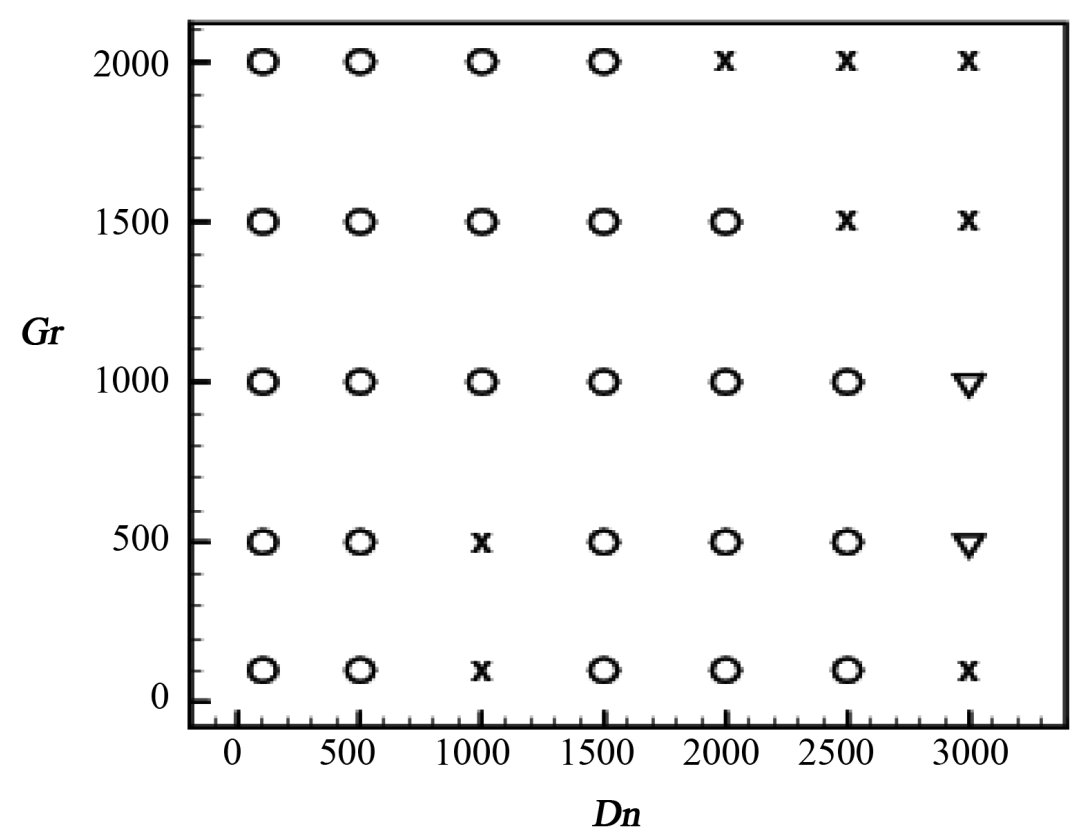

Figure 15. Distribution of the time-dependent solutions in the Dean number vs. Grashof number (Dn-Gr) plane for $100 \leq D n \leq 3000$ and $100 \leq G r \leq 2000$ ( O : steady-state solution, $x$ : periodic solution, $\nabla$ : chaotic solution).

are also obtained, and it is found that periodic or multi-periodic solution oscillates between asymmetric two-, and four-vortex solutions, while for chaotic solution, there exist only asymmetric two-vortex solution. The temperature distribution is consistent with the secondary vortices and it is found that the temperature distribution occurs significantly from the heated wall to the fluid as the secondary flow becomes stronger. The present study also shows that there is a strong interaction between the heating-induced buoyancy force and the centrifugal force in the curved passage which stimulates fluid mixing and thus results in thermal enhancement in the flow.

\section{References}

[1] Dean, W.R. (1927) Note on the Motion of Fluid in a Curved Pipe. Philosophical Magazine, 4, 208-223. http://dx.doi.org/10.1080/14786440708564324

[2] Berger, S.A., Talbot, L. and Yao, L.S. (1983) Flow in Curved Pipes. Annual Review of Fluid Mechanics, 35, 461-512. http://dx.doi.org/10.1146/annurev.fl.15.010183.002333

[3] Nandakumar, K. and Masliyah, J.H. (1986) Swirling Flow and Heat Transfer in Coiled and Twisted Pipes. Advances in Transport Process, 4, 49-112.

[4] Ito, H. (1987) Flow in Curved Pipes. JSME International Journal, 30, 543-552.

[5] Winters, K.H. (1987) A Bifurcation Study of Laminar Flow in a Curved Tube of Rectangular Cross-Section. Journal of Fluid Mechanics, 180, 343-369. http://dx.doi.org/10.1017/S0022112087001848

[6] Daskopoulos, P. and Lenhoff, A.M. (1989) Flow in Curved Ducts: Bifurcation Structure for Stationary Ducts. Journal of Fluid Mechanics, 203, 125-148. http://dx.doi.org/10.1017/S0022112089001400

[7] Mondal, R.N. (2006) Isothermal and Non-Isothermal Flows through Curved Duct with Square and Rectangular CrossSection. Ph.D. Thesis, Department of Mechanical and Systems Engineering, Okayama University, Japan.

[8] Yanase, S. and Nishiyama, K. (1988) On the Bifurcation of Laminar Flows through a Curved Rectangular Tube. Journal of the Physical Society of Japan, 57, 3790-3795. http://dx.doi.org/10.1143/JPSJ.57.3790

[9] Yanase, S., Kaga, Y. and Daikai, R. (2002) Laminar Flow through a Curved Rectangular Duct over a Wide Range of the Aspect Ratio. Fluid Dynamics Research, 31, 151-183. http://dx.doi.org/10.1016/S0169-5983(02)00103-X

[10] Wang, L. and Yang, T. (2005) Periodic Oscillation in Curved Duct Flows. Physica D, 200, 296-302. http://dx.doi.org/10.1016/j.physd.2004.11.003

[11] Wang, L.Q. and Yang, T.L. (2004) Multiplicity and Stability of Convection in Curved Ducts: Review and Progress, 
Advances in Heat Transfer, 38, 203-256. http://dx.doi.org/10.1016/s0065-2717(04)38004-4

[12] Yanase, S., Mondal, R.N., Kaga, Y. and Yamamoto, K. (2005) Transition from Steady to Chaotic States of Isothermal and Non-Isothermal Flows through a Curved Rectangular Duct. Journal of the Physical Society of Japan, 74, 345-358. http://dx.doi.org/10.1143/JPSJ.74.345

[13] Yanase, S., Mondal, R.N. and Kaga, Y. (2005) Numerical Study of Non-Isothermal Flow with Convective Heat Transfer in a Curved Rectangular Duct. International Journal of Thermal Sciences, 44, 1047-1060. http://dx.doi.org/10.1016/j.ijthermalsci.2005.03.013

[14] Mondal, R.N., Kaga, Y., Hyakutake, T. and Yanase, S. (2007) Bifurcation Diagram for Two-Dimensional Steady Flow and Unsteady Solutions in a Curved Square Duct. Fluid Dynamics Research, 39, 413-446. http://dx.doi.org/10.1016/j.fluiddyn.2006.10.001

[15] Mondal, R.N., Uddin M.S. and Yanase, S. (2010) Numerical Prediction of Non-Isothermal Flow through a Curved Square Duct. International Journal of Fluid Mechanics Research, 37, 85-99. http://dx.doi.org/10.1615/InterJFluidMechRes.v37.i1.60

[16] Chandratilleke, T.T. and Nursubyakto, S. (2003) Numerical Prediction of Secondary Flow and Convective Heat Transfer in Externally Heated Curved Rectangular Ducts. International Journal of Thermal Sciences, 42, 187-198. http://dx.doi.org/10.1016/S1290-0729(02)00018-2

[17] Mondal, R.N., Kaga, Y., Hyakutake, T. and Yanase, S. (2006) Effects of Curvature and Convective Heat Transfer in Curved Square Duct Flows. Journal of Fluids Engineering, 128, 1013-1023. http://dx.doi.org/10.1115/1.2236131

[18] Norouzi, M., Kayhani, M.H., Nobari, M.R.H. and Karimi Demneh, M. (2009) Convective Heat Transfer of Viscoelastic Flow in a Curved Duct, World Academy of Science, Engineering and Technology, 32, 327-333.

[19] Norouzi, M., Kayhani, M.H., Shu, C. and Nobari, M.R.H. (2010) Flow of Second-Order Fluid in a Curved Duct with Square Cross-Section. Journal of Non-Newtonian Fluid Mechanics, 165, 323-339. http://dx.doi.org/10.1016/j.jnnfm.2010.01.007

[20] Chandratilleke, T.T., Nadim, N. and Narayanaswamy, R. (2012) Vortex Structure-Based Analysis of Laminar Flow Behaviour and Thermal Characteristics in Curved Ducts. International Journal of Thermal Sciences, 59, 75-86. http://dx.doi.org/10.1016/j.ijthermalsci.2012.04.014

[21] Gottlieb, D. and Orazag, S.A. (1977) Numerical Analysis of Spectral Methods. Society for Industrial and Applied Mathematics, Philadelphia.

[22] Ligrani, P.M. and Niver, R.D. (1988) Flow Visualization of Dean Vortices in a Curved Channel with 40 to 1 Aspect Ratio. Physics of Fluids, 31, 3605. http://dx.doi.org/10.1063/1.866877 